\title{
Radionuclide techniques for myocardial viability and hibernation
}

In hibernation there is a persistent, reversible abnormality of contraction of viable myocardium that normalises upon revascularisation. It may be short term, in which case no ultrastructural changes occur, or long term when myofibrils are disorganised and reduced in number, and myocardial glucose content and extracellular collagen are increased. There is usually some preservation of flow reserve, and hibernating myocardium generally responds to inotropic stimulation with an increase in contraction.

In myocardial stunning there is transient depression of ventricular contraction which is induced by ischaemia and which persists after the ischaemic insult, despite the absence of irreversible damage and the restoration of normal or near normal perfusion.

Although the concepts of hibernation and stunning are superficially distinct, in reality there is considerable overlap. In contrast to earlier expectations which imagined that hibernation was an adaptive response to a chronic reduction of blood flow, recent work has demonstrated that akinetic or severely hypokinetic myocardial segments, which bear all the hallmarks of hibernation and which subsequently have improved contraction after revascularisation, may be associated with normal or near normal perfusion at rest! ${ }^{12}$ Patients with reversible abnormalities of myocardial contraction induced by ischaemia are probably a heterogeneous group in which intermittently or permanently reduced perfusion, recurrent stunning, and long term ultrastructural changes interact to a variable degree. In the face of such heterogeneity, it is probably simplistic to consider that any single imaging approach will be applicable to all patients and all situations, unless it can address all of these variables.

\section{Positron emission tomography}

Many consider positron emission tomography (PET) to be the gold standard for the detection of hibernating myocardium. Using ${ }^{13} \mathrm{~N}$-ammonia $\left({ }^{13} \mathrm{NH}_{3}\right)$ as a marker of perfusion and ${ }^{18} \mathrm{~F}$ fluorodeoxyglucose $\left({ }^{18} \mathrm{FDG}\right)$ as a marker of glucose uptake (and, therefore, metabolic activity), infarcted myocardium is characterised by low uptake of both tracers (a so called matched defect); hibernating myocardium has a disproportionately high glucose uptake relative to flow (mismatched defect). In a number of studies, the extent of mismatch has been shown to predict postrevascularisation improvement of left ventricular contraction $^{3}$ with an accuracy greater than $80 \%$, to predict relief of heart failure symptoms, ${ }^{4}$ and, as there is some evidence to suggest that hibernating myocardium is an unstable substrate, to indicate prognosis. ${ }^{56}$ Although valuable, PET is not perfect. Improved predictive accuracy might be obtained if measures of flow reserve or demonstration of increased contraction after inotropic stimulation were added to conventional ${ }^{13} \mathrm{NH}_{3}{ }^{18} \mathrm{FDG}$ mismatch criteria. Alternatively, novel tracers may furnish additional information. For example, regional decay of radioactivity after uptake of ${ }^{11} \mathrm{C}$ acetate is thought to reflect oxidative metabolism, and rapid exchange of ${ }^{15} \mathrm{O}$ water is thought to be a property only of living myocardium; both of these tracers are currently under evaluation as markers of viability.

Single photon emission computed tomography

PET is expensive and confined to specialist centres. Single photon emission computed tomography (SPECT) is considerably cheaper and widely available. SPECT differs crucially from PET in two ways. First, attenuation correction is an integral component of PET, whereas it is currently in the development stage with SPECT. Therefore, not only are image quality and resolution generally superior with PET, but tracer uptake can be quantified. Second, although there have been attempts to image ${ }^{18}$ FDG with conventional $\gamma$ cameras, the tracers used in SPECT are different, both in terms of uptake mechanisms and distribution, from those used in PET. Accordingly, results from PET cannot be extrapolated directly to SPECT.

When SPECT is used to detect stress induced myocardial ischaemia, ${ }^{99 \mathrm{~m}}$ technetium based tracers have largely displaced ${ }^{201}$ thallium. However, when used to detect myocardial hibernation or viability the experience with such tracers has been disappointing. ${ }^{7-9}$ Certainly, uptake of tracers such as ${ }^{99 \mathrm{~m}}$ technetium-sestamibi seems to imply viability, but the converse does not seem to be the case; many apparent defects on sestamibi SPECT can be shown to take up ${ }^{18} \mathrm{FDG}$ on PET and, therefore, to contain viable muscle. In particular, this seems to be the case with inferior defects; therefore, it may be a problem with attenuation, but it cannot be ruled out that fundamental properties of the tracer with respect to uptake and retention are also involved.

Initial experience with ${ }^{201}$ thallium was not encouraging. When used to detect stress induced ischaemia, a stress-redistribution protocol is usual. Tracer injection is made at peak exercise or immediately after drug administration. Defects on early images reflect both stress induced ischaemia and old infarction. Over the next few hours, thallium redistributes into ischaemic but not infarcted segments. A second set of images taken at four hours-the redistribution images - can then be compared with the original stress images. Filling in of any early defects reliably identifies stress induced ischaemia, but initial comparisons with PET revealed that there was ${ }^{18} \mathrm{FDG}$ uptake in up to $50 \%$ of persistent defects on the redistribution scintigram. ${ }^{10} 11$ Thus, a fixed defect at redistribution underestimates myocardial viability. When there is a further injection of tracer immediately after the redistribution images however (a stress-redistribution-reinjection protocol) much improved results can be obtained and this refined approach has been widely adopted. ${ }^{12}$

In this issue, Yamagishi et al report the results of a study in which stress-redistribution, ${ }^{201}$ thallium SPECT data are combined with ECG results - in particular, whether ST segment elevation occurs on exercise. ${ }^{13}$ They apply this technique to a group of patients early after myocardial 
infarction, using PET ${ }^{18} \mathrm{FDG} /{ }^{13} \mathrm{NH}_{3}$ mismatch as the arbiter of hibernation. The results suggest that, when attempting to identify hibernating myocardium, adding ECG data to SPECT data produces improved accuracy compared to either alone, especially in anterior infarction. There are, however, a number of caveats. Not only do they introduce a new technique, but they apply it to a group of patients early after myocardial infarction, in whom the balance between stunning and classic hibernation may well differ from that in subjects with more remote infarction (numbers are small). Furthermore, few would use a redistribution only approach to identify hibernating myocardium with ${ }^{201}$ thallium; is it better to add in ECG data, to reinject ${ }^{201}$ thallium or to do both? Finally, although PET is a good indicator of viability, the proof of any new approach must be prediction of improved contraction after revascularisation, improvement in symptoms or increased longevity.

Cardiac Department,

STEPHEN WALTON

Aberdeen Royal Infirmary,

Foresterhill, Aberdeen AB25 2ZN, UK

1 Conversano A, Walsh JF, Geltman, et al. Delineation of myocardial stunning and hibernation by positron emission tomography in advanced coronary artery disease. Am Heart f 1996;131:440-50

2 Gerber BL, Vanoverschelde JL, Bol A, et al. Myocardial blood flow, glucose uptake and recruitment for inotropic reserve in chronic left ventricular ischaemic dysfunction. Implications for the pathophysiology of chronic myocardial hibernation. Circulation 1996:94:651-9.
3 Tillisch J, Brunken R, Marshall R, et al. Reversibility of cardiac wall motion abnormality predicted by positron emission tomography. $N$ Engl f Med 1986;34:884-8.

4 DiCarli MF, Asgarzadie F, Schelbert HR, et al. Quantitative relation between myocardial viability and improvement in heart failure symptoms after revascularisation in patients with ischemic cardiomyopathy. Circulation 1995;92:3436-44.

5 Eitzman D, Al-Aouar Z, Kanten H, et al. Clinical outcome of patients with advanced coronary artery disease after viability studies with positron emission tomography. F Am Coll Cardiol. 1992;20:559-65.

6 DiCarli MF, Davidson M, Little R, et al. Value of metabolic imaging with positron emission tomography for evaluating prognosis in patients with coronary artery disease and left ventricular dysfunction. Am $\mathcal{f}$ Cardiol 1994;73:527-33.

7 Altehoefer C, Hens-Jurgen K, Dorr R, et al. Fluorine-18 deoxyglucose PET for assessment of viable myocardium in perfusion defects in Tc $99 \mathrm{~m}$ MIBI SPECT: a comparative study in patients with coronary artery disease. Eur f Nucl Med 1992;19:334-42.

8 Sawada SG, Allman KC, Muzik O, et al. Positron emission tomography detects evidence of viability in rest technetium $99 \mathrm{~m}$ sestamibi defects. $\mathcal{F} \mathrm{Am}$ Coll Cardiol 1994;23:92-8.

9 Soufer R, Dey HM, Ng CK, et al. Comparison of sestamibi single photon computed tomography to positron emission tomography for estimating left ventricular myocardial viability. Am f Cardiol 1995;75:1214-19.

10 Brunken R, Schwaiger M, Grover-McKay M, et al. Positron emission tomography detects tissue metabolic activity in myocardial segments with persistent thallium perfusion defects at Tl-201 SPECT. 7 Am Coll Cardiol 1987; 10:557-67.

11 Brunken R, Kottau S, Nienabar CA, et al. PET detection of viable tissue in myocardial segments with perfusion defects at Tl-201 SPECT. Radiology 1989;172:65-73.

12 Dilsizian, V, Rocco TP, Freedman NMT, et al. Enhanced detection of ischaemic but viable myocardium by the re-injection of thallium after stress-redistribution studies N Eng 7 Med 1990;323:141-6.

13 Yamagishi H, Akioka K, Takagi M, et al. Exercise four hour redistribution thallium-201 single photon emission computed tomography and exercise induced ST segment elevation in detecting the viable myocardium in patients with acute myocardial infarction. Heart 1999;81:17-24. 